OPEN ACCESS

Edited by:

Xuezhou Zhou,

Beijing Jiaotong University, China

Reviewed by:

Lei Chen,

Fujian Agriculture and Forestry

University, China

Priyia Pusparajah,

Monash University Malaysia, Malaysia

*Correspondence:

Jianhua Zhang

petermails@zzu.edu.cn

Xiaobo Mao

mail-mxb@zzu.edu.cn

Chuanliang Chen

henanccl@163.com

Specialty section: This article was submitted to

Ethnopharmacology,

a section of the journal

Frontiers in Pharmacology

Received: 25 December 2020

Accepted: 24 February 2021

Published: 07 April 2021

Citation:

Wang Z, Cheng L, Shang Z, Li Z, Zhao $Y$, Jin W, Li Y, SU F, Mao $X$, Chen $C$ and Zhang J (2021) Network Pharmacology for Analyzing the Key Targets and Potential Mechanism of Wogonin in Gliomas.

Front. Pharmacol. 12:646187. doi: 10.3389/fphar.2021.646187

\section{Network Pharmacology for Analyzing the Key Targets and Potential Mechanism of Wogonin in Gliomas}

\author{
Zaizhong Wang ${ }^{1,2}$, Lulu Cheng ${ }^{3}$, Zhigang Shang ${ }^{4}$, Zhihui Li $^{4}$, Yuping Zhao ${ }^{5}$, Wenwen Jin ${ }^{1}$, \\ Yingyue $\mathrm{Li}^{1}$, Fangchu Su${ }^{1}$, Xiaobo Mao ${ }^{4 *}$, Chuanliang $\mathrm{Chen}^{6 *}$ and Jianhua Zhang ${ }^{1 *}$ \\ ${ }^{1}$ Medical Engineering Technology and Data Mining Institute, Zhengzhou University, Zhengzhou, China, ${ }^{2}$ Department of \\ Neurosurgery, Zhumadian Central Hospital, Zhumadian, China, ${ }^{3}$ Digital Medical Laboratory, Zhumadian Central Hospital, \\ Zhumadian, China, ${ }^{4}$ School of Electrical Engineering, Zhengzhou University, Zhengzhou, China, ${ }^{5}$ Technology Department, China \\ Academy of Chinese Medical Sciences, Beijing, China, ${ }^{6}$ Clinical Bioinformatics Experimental Center, Henan Provincial People's \\ Hospital, Zhengzhou, China
}

Objective: To analyze the key targets and potential mechanisms underlying the volatile components of Scutellaria baicalensis Georgi acting on gliomas through network pharmacology combined with biological experiments.

Methods: We have extracted the volatile components of Scutellaria baicalensis by gas chromatography-mass spectrometry (GC-MS) and determined the active components related to the onset and development of gliomas by combining the results with the data from the Traditional Chinese Medicine Systems Pharmacology database. We screened the same targets for the extracted active components and gliomas through network pharmacology and then constructed a protein-protein interaction network. Using a Gene Ontology and Kyoto Encyclopedia of Genes and Genomes (KEGG) analysis, we analyzed the protein effects and regulatory pathways of the common targets. Lastly, we employed ELISA and Western blot in verifying the key targets in the regulatory pathway.

Results: We ultimately determined that the active component in S. baicalensis Georgi related to the onset and development of gliomas was Wogonin. The results of the network pharmacology revealed 85 targets for glioma and Wogonin. We used gene ontology to analyze these target genes and found that they involved 30 functions, such as phosphatidylinositol phosphokinase activation, while the KEGG analysis showed that there were 10 regulatory pathways involved. Through the following analysis, we found that most of the key target genes are distributed in the PI3K-Akt and interleukin 17 signaling pathways. We then cultured U251 glioma cells for the experiments. Compared with the control group, no significant change was noted in the caspase-3 expression; however, cleaved caspase-3 expression increased significantly and was dosedependent on Wogonin. The expression of Bad and Bcl-2 with $25 \mu \mathrm{M}$ of Wogonin has remained unchanged, but when the Wogonin dose was increased to $100 \mu \mathrm{M}$, the expression of Bad and Bcl-2 was noted to change significantly (Bad was significantly upregulated, while $\mathrm{Bcl}-2$ was significantly downregulated) and was dose-dependent on Wogonin. The ELISA results showed that, compared with the control group, the 
secretion of tumor necrosis factor alpha, IL-1 $\beta$, and IL-6 decreased as the Wogonin concentration increased. Tumor necrosis factor alpha downregulation had no significant dose-dependent effect on Wogonin, the inhibitory effect of $25 \mu \mathrm{M}$ of Wogonin on IL-6 was not significant, and IL-1 $\beta$ downregulation had a significant dose-dependent effect on Wogonin.

Conclusion: Wogonin might promote the apoptosis of glioma cells by upregulating proapoptotic factors, downregulating antiapoptotic factors, and inhibiting the inflammatory response, thereby inhibiting glioma progression.

Keywords: glioma, wogonin, network pharmacology, signal path, key target

\section{INTRODUCTION}

Glioblastoma multiforme, also called glioma, is a relatively common malignant brain tumor in adults, which originates from glial cells, is a highly invasive and fatal tumor of the central nervous system (Galldiks et al., 2017), and it is not only prone to relapse but also has a high mortality rate. Relevant studies have shown that the mean survival time for glioma is 6-14 months, which represents a serious threat to the life and health of such patients (Aquino et al., 2017; Wesseling and Capper, 2018). At present, surgical resection combined with postoperative chemoradiotherapy is the main treatment for glioma. Due to issues such as the immunosuppression caused by surgery and chemoradiotherapy, residual tumor cells can grow rapidly in a short period and thereby cause a relapse (Hervey-Jumper and Berger, 2019). Some patients also develop resistance to chemotherapy drugs when taken long term, thereby reducing their therapeutic effect.

Natural products are an abundant treasure trove for the study of and search for active drugs against glioma and other cancers. The use of traditional Chinese medicine (TCM) in order to assist in the treatment of glioma has raised interest. TCM can improve the clinical symptoms of various types of glioma, reduce the adverse reactions after chemotherapy, enhance immunity, and prolong survival times (Scheck et al., 2006). Current research is therefore focused on finding glioma treatment drugs from natural TCM. Radix scutellariae, the root of the S. baicalensis Georgi (Chinese skullcap) plant, has been determined to have numerous pharmacological activities in TCM, including antiinflammatory effects, which are particularly important, in addition, This TCM has an antiapoptotic effect and is currently employed in treating gliomas, although more research is needed. One of the active ingredients of $S$. baicalensis Georgi that plays a role in TCM is the flavonoid baicalin, which has a significant effect in the treatment of gliomas (Kyo et al., 1998; Lee et al., 2005; Zhang et al., 2014); however, there has been less research on the volatile components of this plant.

Therefore, to examine the effects of the volatile components of $S$. baicalensis on gliomas, we extracted the volatile components of $S$. baicalensis by gas chromatography-mass spectrometry (GC-MS) and then searched and matched the active components related to the onset and development of glioma from the Traditional Chinese Medicine Systems Pharmacology (TCMSP) database. Employing the network pharmacology method, we screened the possible pathways of the involvement of $S$. baicalensis Georgi in the onset and development of glioma and further verified and analyzed the key signal nodes through biological experiments to reveal the potential mechanisms of the volatile components of this plant in the treatment and prevention of gliomas.

\section{MATERIALS AND METHODS}

\section{Extraction of Volatile Components from Scutellaria baicalensis Geologia by Gas Chromatography-Mass Spectrometry}

\section{Sample Preparation}

We crushed and sipped $30 \mathrm{~g}$ of Scutellaria and divided the result into three equal parts ( $10 \mathrm{~g}$ each), which we later immersed in hot water for $4 \mathrm{~h}$ with the ethanol $\left(81.5^{\circ} \mathrm{C}\right)$, methanol $\left(64.7^{\circ} \mathrm{C}\right)$, and benzenol $(71 \mathrm{C})$, respectively. After passing the resulting liquid through a $0.22-\mu \mathrm{m}$ ultrafiltration membrane, the filtrate was concentrated to $5 \mathrm{ml}$ using a rotary evaporator and filtered through a $0.22-\mu \mathrm{m}$ filter membrane into a sample injection bottle, which we then analyzed using GC-MS as detailed below.

\section{Gas Chromatography Conditions}

We heated an RTX-5MS $(30 \mathrm{~m} \times 0.25 \mathrm{~mm} \times 0.25 \mu \mathrm{m})$ elastic quartz capillary column using a heating program. The column temperature was raised to $210^{\circ} \mathrm{C}$ at a rate of $10^{\circ} \mathrm{C} / \mathrm{min}$, then to $220^{\circ} \mathrm{C}$ at $1{ }^{\circ} \mathrm{C} / \mathrm{min}$, and then to $280{ }^{\circ} \mathrm{C}$ at $10^{\circ} \mathrm{C} / \mathrm{min}$. The temperature was then kept steady for $1 \mathrm{~min}$. The total determination time was $20 \mathrm{~min}$, the shunt ratio was $20: 1$, the inlet temperature was $280^{\circ} \mathrm{C}$, the column front pressure was $50 \mathrm{kPa}$, the injection volume was $1 \mu \mathrm{L}$, the carrier gas was high purity nitrogen $(99.999 \%)$, and the flow rate was $1.1 \mathrm{ml} / \mathrm{min}$. 


\section{Mass Spectrum Conditions}

The settings were as follows: electron ionizationion source; ion source temperature of $200^{\circ} \mathrm{C}$; electron energy of $70 \mathrm{eV}$; connector temperature of $250^{\circ} \mathrm{C}$; solvent delay of $3.5 \mathrm{~min}$; scanning range: 50-600 amu; and electron multiplier voltage of $1200 \mathrm{~V}$.

\section{Screening with the Prior Data}

For the screening, we generated the corresponding compound ion flow chart, based on the mass spectrum of each chromatographic peak fragment figure, the literature review, and the database data. We also checked the mass spectrum data on the base peak's mass-to-charge ratio and the relative abundance condition as the intuitive comparison. Finally, we determined the crude drug's chemical composition and its physical and chemical properties and pharmacological action.

\section{Screening and Identifying the Volatile Active Components of Scutellaria}

The active components of the GC-MS S. baicalensis extracts were screened using the TCMSP database under conditions of oral bioavailability of $30 \%$, druggability of 0.18 , and bloodbrain barrier (BBB) permeability index of -0.3 . We downloaded the chemical structure files corresponding to the active components.

\section{Construction of the Volatile Chemical Composition Library and Target Prediction}

We then determined the specific chemical information for Wogonin (a flavonoid-like chemical compound found in $S$. baicalensis) using the PubChem database. We predicted the target genes using BATMAN-TCM combined with the webbased SwissTargetPrediction tool. Lastly, we selected the target protein name, gene name, UniProt ID, and other information.

\section{Disease-Related Target Analysis}

We searched the DisGeNET NCBI-gene and GeneCards databases using the keyword "glioma" and obtained the target GENE-related data for glioma regulation. We employed UniProt to improve the target's UniProt ID and gene name.

\section{Gene Ontology and Kyoto Encyclopedia of Genes and Genomes Enrichment Analysis}

We entered the retrieved target genes into the Cytoscape software and employed and Gene Ontology (GO) and Kyoto Encyclopedia of Genes and Genomes (KEGG) in the ClueGo plug-in for the biological process analysis and pathway analysis of the TCM volatile components acting on glioma-related targets. The parameters were set as ClueGo: Function, GO and KEGG, and Use GO Term Fusion ( $\mathrm{p} \leq 0.05)$.

\section{Construction of the Volatile Component-Target-Disease Pathway for Traditional Chinese Medicine}

As per the established Wogonin and disease-related targets, we screened out the common targets of drugs and diseases and constructed the disease-target regulation network of volatile component action targets and glioma-related diseases of Genuine medicinal materials in Henan using the Search Tool for the Retrieval of Interacting Genes/ Proteins (STRING) database. We then inserted the acquired volatile components, key targets of compounds, and main pathways for TCM into Cytoscape software, constructing the glioma-related component-target-main disease pathways using Cytoscape's Merge and Union functions.

\section{Experimental Verification of Wogonin on Inflammatory Factors and Apoptotic-Related Proteins in Gliomas}

We cultured human U251 cells and treated them with $0,25,100$, and $200 \mu \mathrm{M}$ of Wogonin (HPLC $\geq 98 \%$, standard) for $24 \mathrm{~h}$, WOG concentration was based on reference (Wang et al., 2013). we used ELISA to analyze the expression levels of inflammatory factors IL-1, IL-6 and tumor necrosis factor alpha (TNF- $\alpha$ ) in the cell supernatant by Western blot analysis (four groups, three indicators in total). We used ELISA to detect the expression of apoptosis-related protein caspase-3/cleaved caspase-3 Bcl2 Bad.

\section{Cell Culture}

We cultured U251 cells with 10\% FBS supplemented DMEM in an incubator set to $375 \% \mathrm{CO}_{2}$. These cells are obtained from the laboratory. We then grouped the cells as follows: control group $(0 \mu \mathrm{M}), 25 \mu \mathrm{M}, 100 \mu \mathrm{M}$, and $200 \mu \mathrm{M}$.

\section{ELISA}

We used ELISA kit (Wanleibio Co., Ltd.) to detect the expression of TNF- $\alpha$, IL- $1 \beta$ and IL- 6 according to the instructions of the kit.

\section{Western Blot}

1) After the cells were lyzed, the protein in it was extracted, and the concentration of the above-mentioned protein was determined using a measurement kit (Wanleibio Co., Ltd. Shenyang, China).

2) We used polyacrylamide gel to extract the protein of each lysate $(20 \mu \mathrm{g})$, and then transferred it to a polyvinylidene fluoride membrane (Millipore, Bedford, MA, United States).

3) The above membrane was washed with $0.5 \%$ Tween 20 (TBST) in Tris buffered saline for $5 \mathrm{~min}$, and then blocked in 5\% skim milk for $1 \mathrm{~h}$.

4) The membranes were incubated with antibodies against caspase3/cleavedcaspase-3, BAD, and Bcl-2 (Wanleibio 


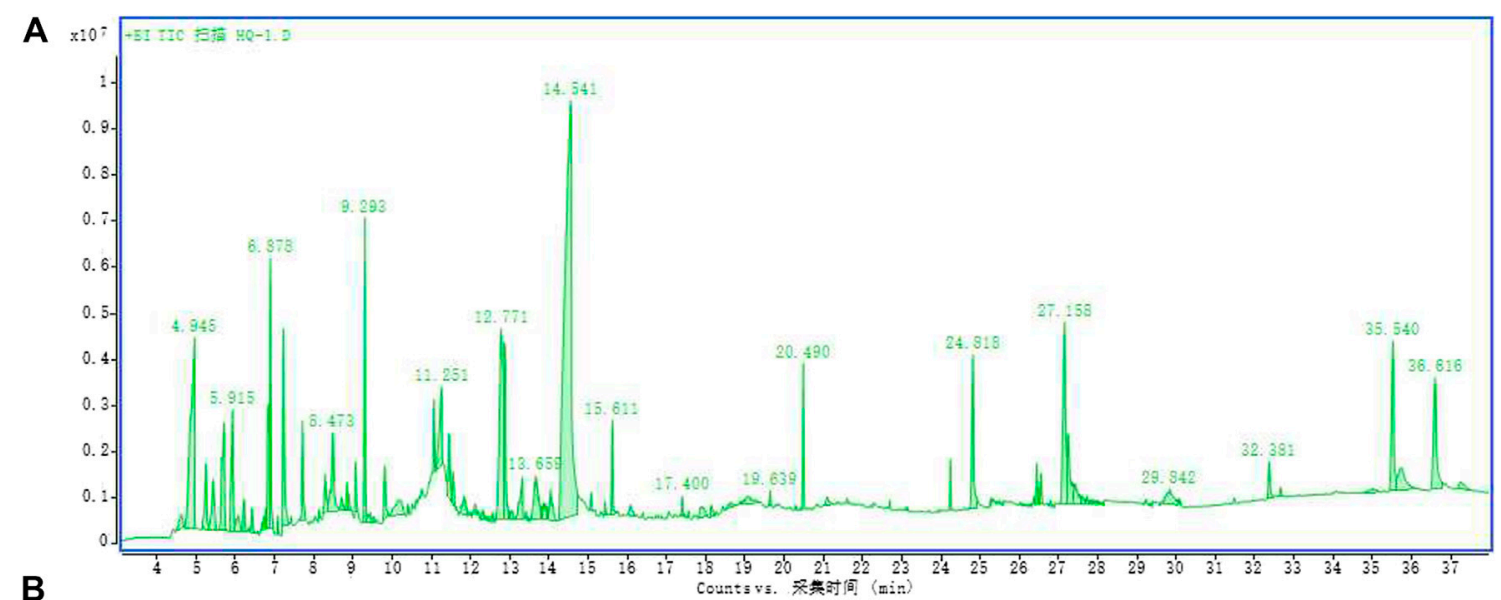

B

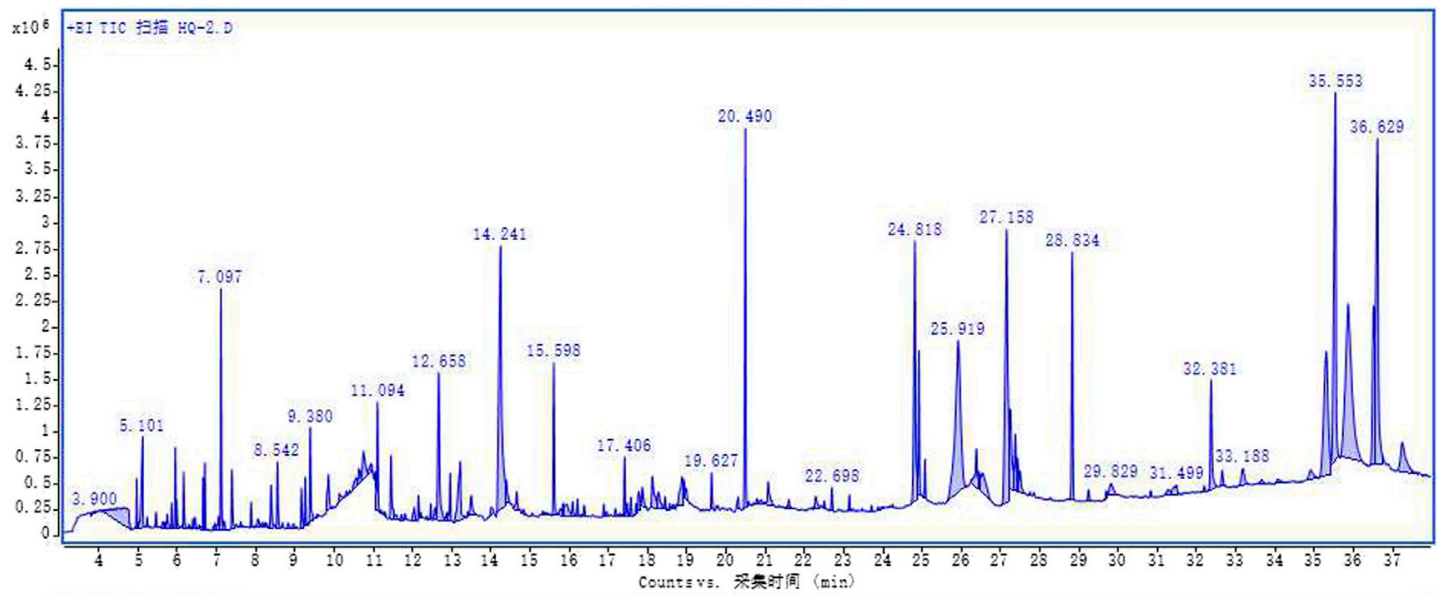

C

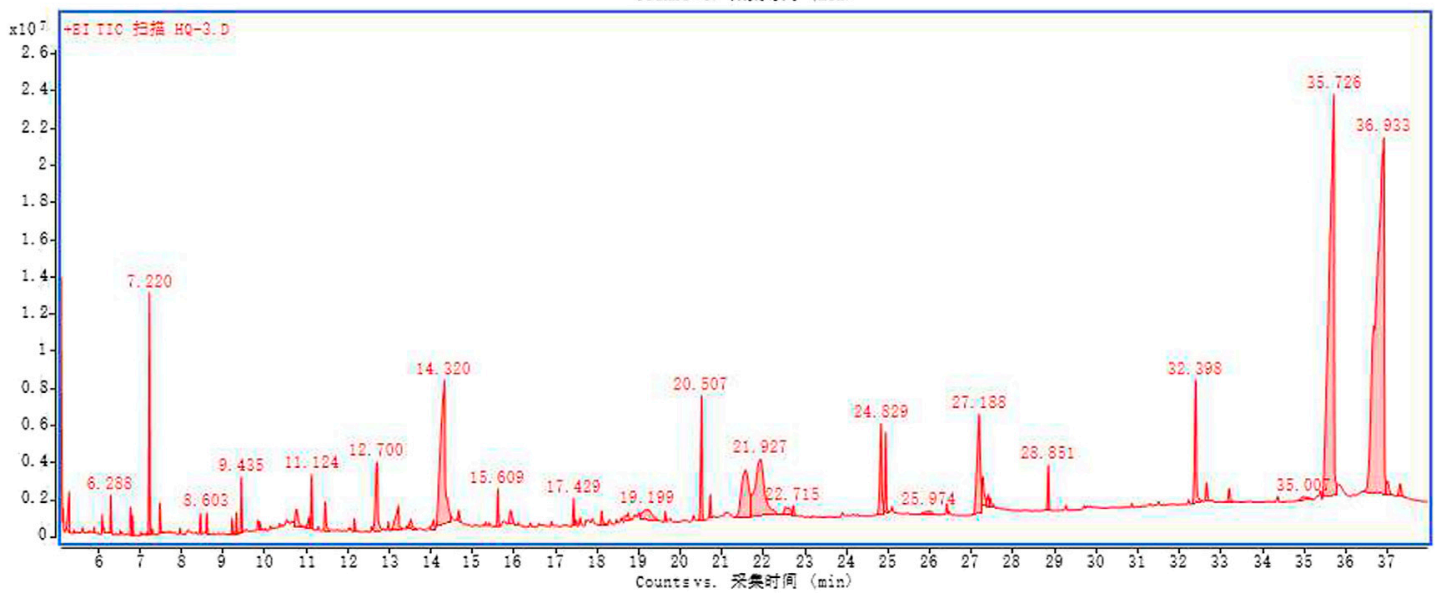

FIGURE 1 | lon flow diagram of the three gas chromatography-mass spectrometry extraction methods for S. baicalensis Georgi by (A) ethanol (B) methanol, and (C) benzyl alcohol.

Co., Ltd. Shenyang, China, 1:2000 dilution) overnight at $4^{\circ} \mathrm{C}$.

5) Successively thereafter, the membranes were washed three times with TBST for $5 \mathrm{~min}$ each, incubated for $1 \mathrm{~h}$ at $37^{\circ} \mathrm{C}$ with rabbit HRP-conjugated antimouse antibody $(1: 1,000)$
(Wanleibio Co., Ltd. Shenyang, China), and washed three times with TBST for 5 min each.

6) We used electrochemiluminescence reagent (Wanleibio Co., Ltd. Shenyang, China) to detect the protein bands. $\beta$-actin was used to normalize the band density. 


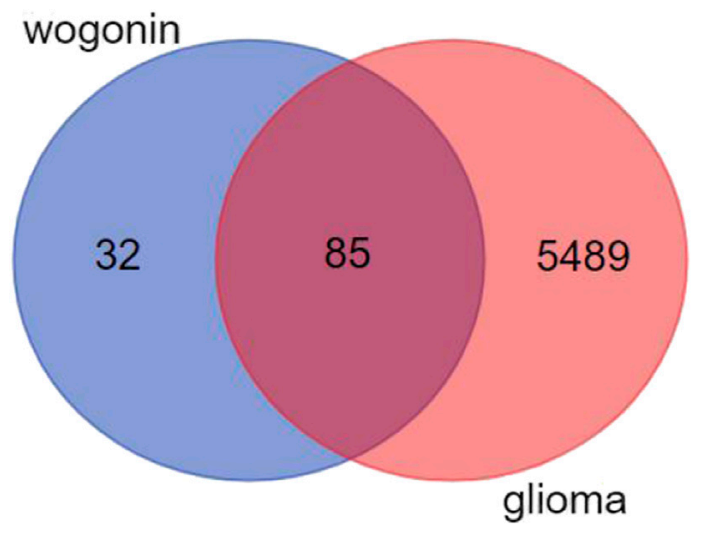

FIGURE 2 | Venn diagram of drug targets and disease proteins.

\section{Statistical Methods}

The statistical analysis was performed using the SPSS v. 23.0 software package, with the measurement data expressed as (x S). We then calculated the differences between groups using a one-way ANOVA, with $p<0.05$ indicating statistically significant differences.

\section{RESULTS}

\section{Extraction of Volatile Components from Scutellaria baicalensis by Gas Chromatography-Mass Spectrometry}

We used the methanol-ethanol-cresol method to extract the volatile active ingredients of $S$. baicalensis by GC-MS. Figures 1, 2 provide the results of the ion flow diagram. The substances related to the onset and development of gliomas were then searched for and matched on the TCMSP database. We finally determined the volatile active ingredient of Scutellaria, that is Wogonin.

\section{Network Analysis of Targets}

In total, 5,574 glioma-related targets were retrieved from the GeneCards, DisGeNET database, and NCBI GENE databases. The Bioinformatics Analysis Tool for the Molecular Mechanism of TCM (BATMAN-TCM) combined with the SwissTargetPrediction tool was able to predict the target genes of the volatile components of $S$. baicalensis, obtaining information on 117 common targets, with 85 combined targets for glioma and Wogonin (Figure 2). We employed Cytoscape software to construct the regulatory network of Wogonin acting on glioma (Figure 3).

\section{Functional Annotation and Kyoto Encyclopedia of Genes and Genomes Pathway Analysis of Glioma Genes}

We employed the clusterProfiler package of $\mathrm{R}$ language to perform the GO annotation and KEGG pathway analysis on
85 target genes of Wogonin acting on gliomas. The GO function annotation analysis results showed that these target genes were involved in the binding of phosphatidylinositol phosphokinase-activating chemokines, L-amino acid translocation, amino acid translocation, and reverse translocation (Figure 4A). The KEGG analysis showed that the regulatory pathway included the PI3K-Akt signaling pathway, the Rap1 signaling pathway, and the IL-17 signaling pathway. These results suggest thatWogonin might affect gliomas via these regulatory pathways (Figure $4 \mathrm{~B}$ ).

The next analysis showed that most of the key target genes are distributed in the PI3K-Akt signaling pathway (an important signaling pathway for tumor apoptosis) and IL-17 signaling pathway (an important inflammatory pathway) (Figure 5). We then selected apoptotic factors such as caspase-3, Bad, and Bcl-2 and inflammatory factors such as IL-1, TNF- $\alpha$, and IL-6 for further experimental verification.

\section{Experimental Verification}

The secretion of TNF- $\alpha$, IL- $1 \beta$, and IL- 6 was detected via ELISA. The groups with Wogonin have showed a decreasing trend in TNF- $\alpha$, IL- $1 \beta$, and IL- 6 secretion with increased Wogonin concentrations compared with the control group (Table 1). Furthermore, TNF- $\alpha$ downregulation was not significantly dose-dependent on Wogonin (Figure 6A), the inhibitory effect of $25 \mathrm{M}$ of Wogonin on IL-6 was not significant (Figure 6B), and IL-1 $\beta$ downregulation was dose-dependent on Wogonin, with significant differences (Figure 6C).

We detected the expression of apoptotic regulation genes such as caspase-3/cleaved caspase-3, Bad, and Bcl-2 by Western blotting (Figure 7A). The results showed that the expression of caspase- 3 in the experimental group was not significantly different from the control group (Figure 7B), in which cleaved caspase-3 expression was significantly increased and was dosedependent on Wogonin (Figure 7C). The expression of Bad and Bcl-2 with 25- $\mu \mathrm{M}$ Wogonin has also remained unchanged; however, when the Wogonin dose was increased to $100 \mu \mathrm{M}$, the expression of $\mathrm{Bad}$ and $\mathrm{Bcl}-2$ was significantly changed (Bad was significantly upregulated, while Bcl-2 was significantly decreased) and was dose-dependent on Wogonin (Figures 7D,E). The relative protein expression of each group was shown in Table 2 . In summary, the above results indicate that Wogonin can effectively reduce the apoptosis of glioma cells by regulating the expression of related proteins, which in turn proves the results of the functional enrichment analysis.

\section{DISCUSSION}

In recent years, extracting effective antitumor components with low toxic and adverse effects from TCM and natural plants has become a new line of research. TCM currently employs mostly boiling water processing in extracting the components of hydrothermally soluble substances in TCM materials. The pharmacological effects of non-hydrothermally soluble substances contained in TCM have gradually gained the attention of researchers (Tu, 2011). Other volatile components 


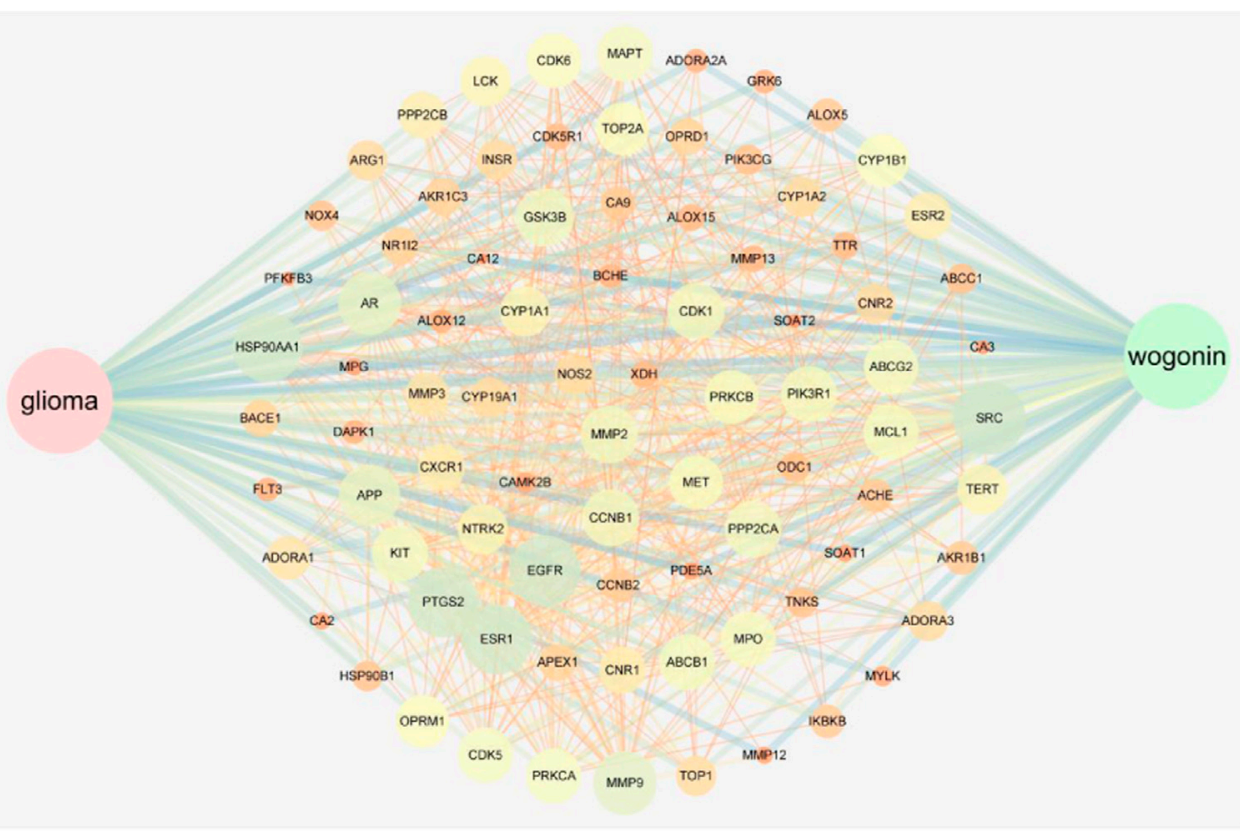

FIGURE 3 | Network analysis of targets compound-target-glioma network.
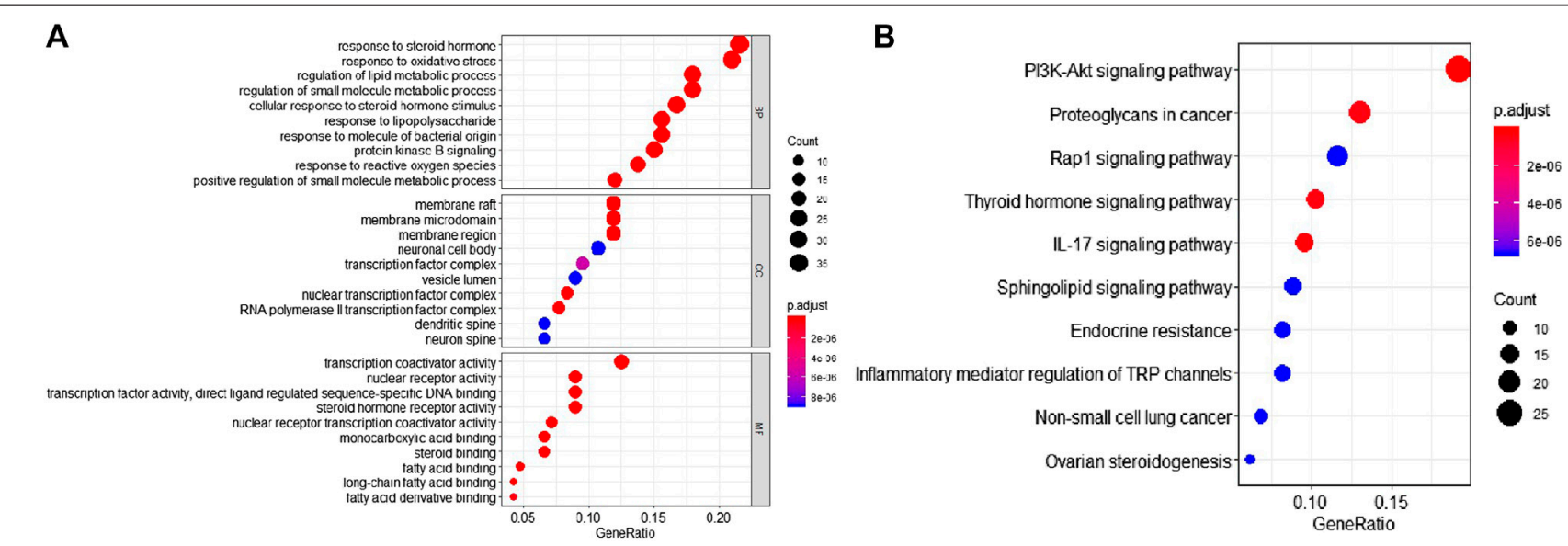

FIGURE 4 | Bioinformatics analysis of drug-disease intersection proteins (A) GO annotations (B) KEGG annotation.

of TCM are currently being analyzed and applied in the prevention and treatment of tumors (Valentovic, 2018). It has been established that the volatile components of TCM have low molecular weight and strong lipid solubility, and can change the ultrastructure of the biofilm barrier in a variety of ways, including inhibiting P-glycoprotein, so that it can easily pass through the $\mathrm{BBB}$, regulate the permeability of the $\mathrm{BBB}$, andenhance brain targeting and affect neurotransmitter content (Fan et al., 2015). In view of the complexity of the volatile components of TCM, the most critical step in their use is screening the content of the different types of volatile components and classifying their efficacy. In this study, we fully extracted the volatile active components of $S$. baicalensis Georgi (methanol, ethanol, and benzenol) and analyzed the components with GC-MS. We then searched and matched those components related to the onset and development of glioma using the TCMSP database. Lastly, we identified the volatile active component Wogonin by analyzing the results of the GC-MS.

Although TCM has been gaining attention worldwide, understanding the scientific basis of TCM from the molecular and systemic level is still a major challenge for evidence-based medicine ( $\mathrm{Li}$ et al., 2017; Sidders et al., 2018). Unlike most modern medical approaches, the role of TCM and its compounds in disease prevention and treatment lies in the overall complex community relationship between the numerous 


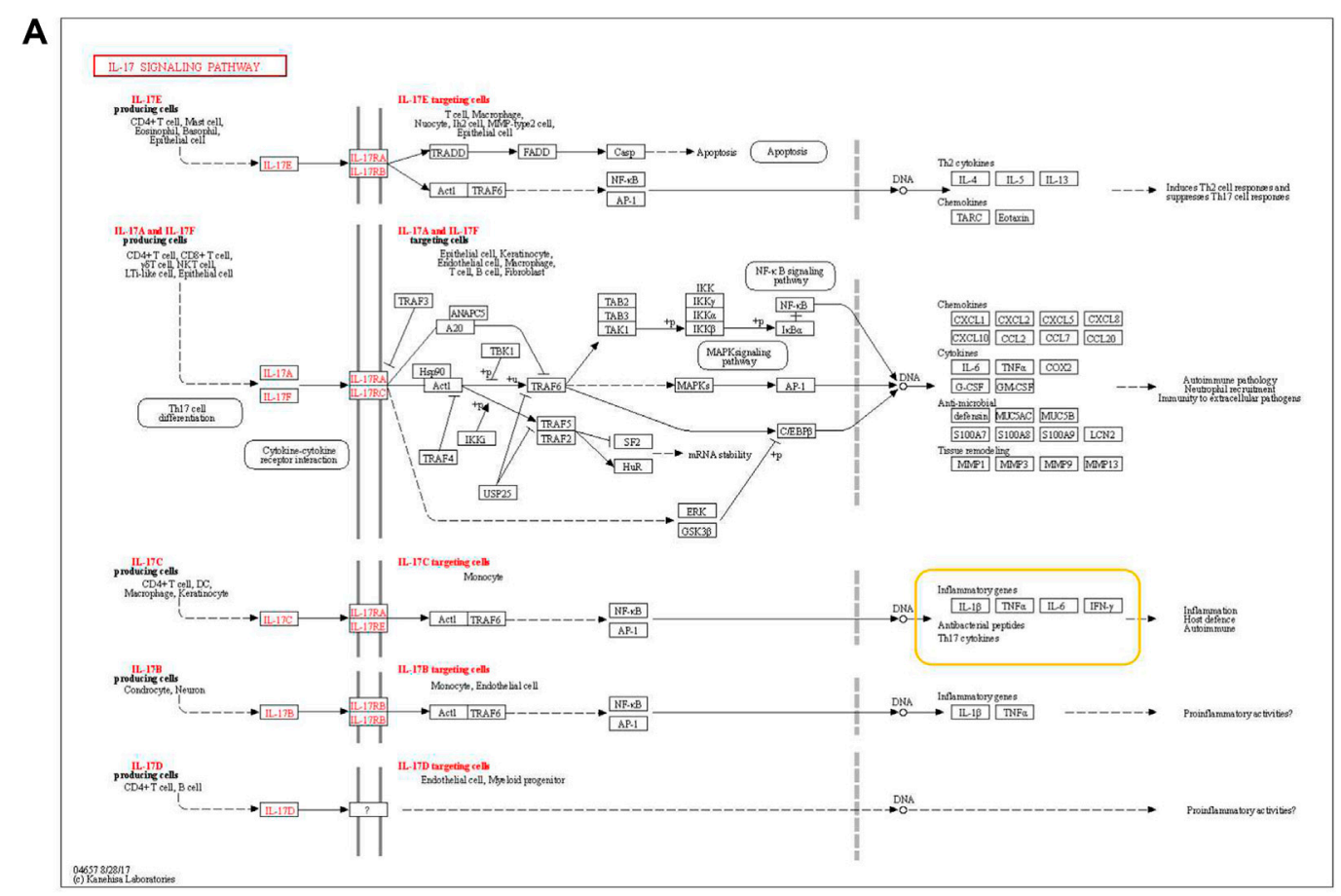

B

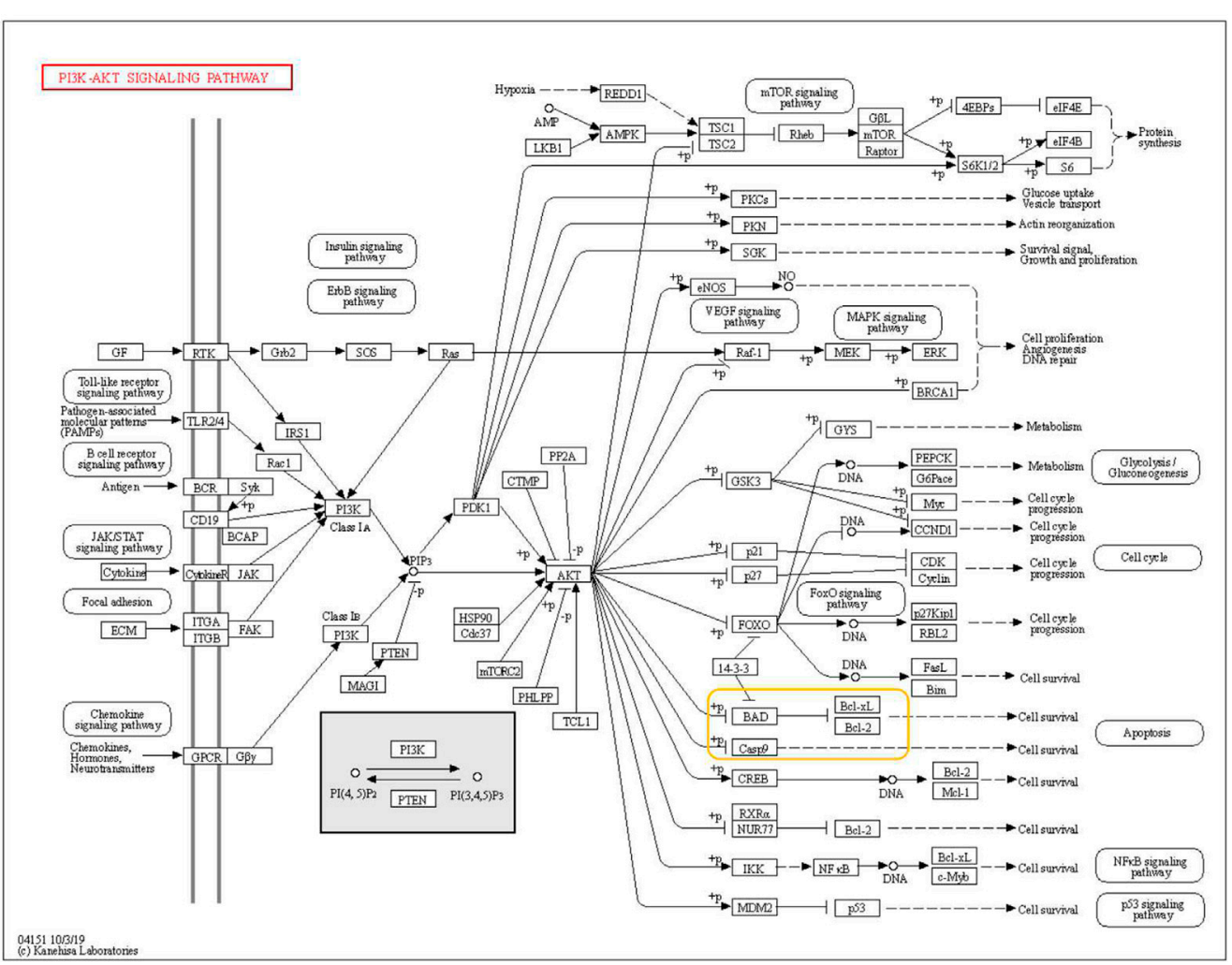

FIGURE 5 | Pi3K-Akt and IL-17 signaling pathway (A) PI3K-Akt signaling pathway and (B) IL-17 signaling pathway, with key target genes in the yellow box.

components, targets, pathways, and functions (Poornima et al., 2016). Network pharmacology employs biological networks as targets to analyze the predictable and systematic relationship between drug targets and diseases in biological networks (Jain et al., 2018). With the rapid development of bioinformatics and pharmacology, the development of network-based drugs has been recognized as a more cost-effective approach to drug development (Jain et al., 2018). 
A

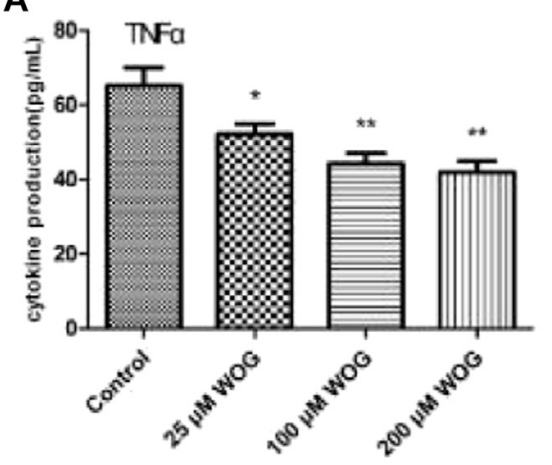

C

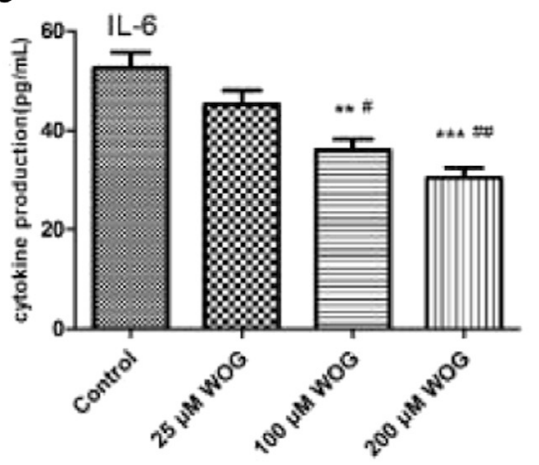

B

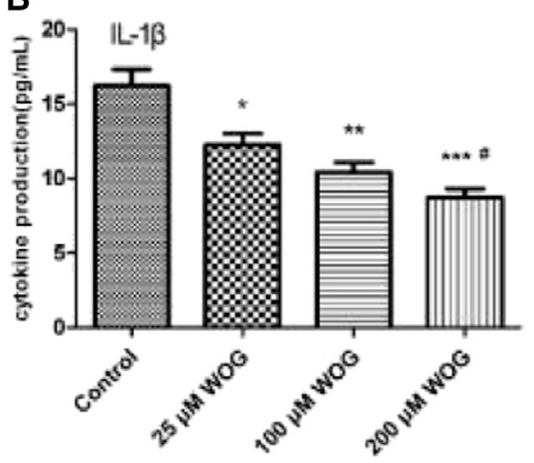

FIGURE 6 | The expression of TNF- $\alpha$ (A), IL-1 $\beta$ (B), and IL-6 (C) was inhibited by Wogonin in U251 cells.

A

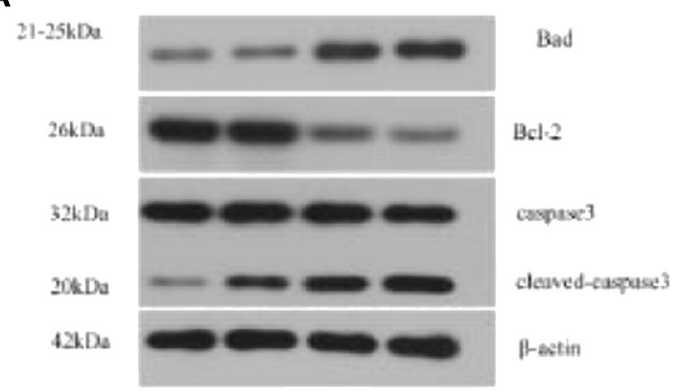

C

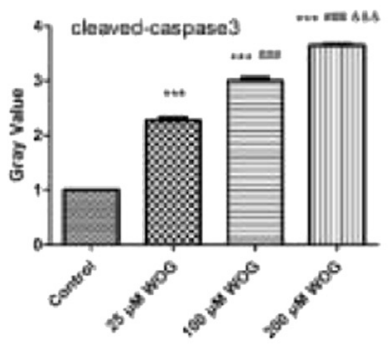

B

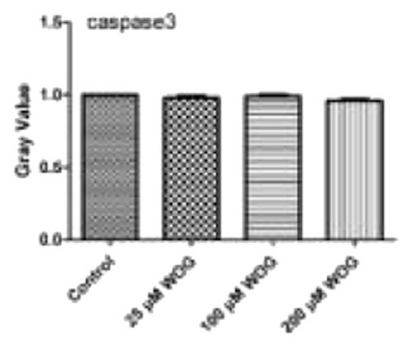

E

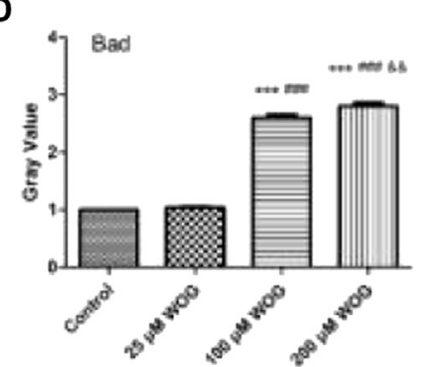

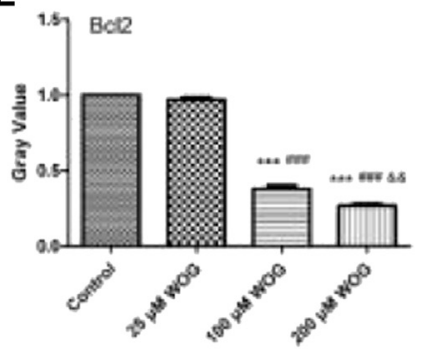

FIGURE 7 | The expression of caspase-3/cleaved-caspase-3, Bad, and Bcl-2 proteins was inhibited by Wogonin in U251 cells (A) Western blot analysis (B-E) Gray analysis. 
TABLE 1 | Wogonin inhibition of the expression of tumor necrosis factor alpha, interleukin-1 $\beta$, and interleukin-6 in the U251 cells.

\begin{tabular}{lccl}
\hline Groups & TNF- $\boldsymbol{\alpha}$ & IL-1 $\boldsymbol{\beta}$ & IL-6 \\
\hline Control group & $65.173 \pm 8.257$ & $16.201 \pm 1.921$ & $52.598 \pm 5.457$ \\
$25 \mu \mathrm{M}$ & $52.093 \pm 4.917^{\mathrm{a}}$ & $12.240 \pm 1.323^{\mathrm{a}}$ & $45.182 \pm 4.889$ \\
$100 \mu \mathrm{M}$ & $44.263 \pm 4.723^{\mathrm{b}}$ & $10.393 \pm 1.173^{\mathrm{b}}$ & $36.002 \pm 3.874^{\mathrm{b}, \mathrm{d}}$ \\
$200 \mu \mathrm{M}$ & $41.938 \pm 4.995^{\mathrm{b}}$ & $8.727 \pm 0.966^{\mathrm{c}, \mathrm{d}}$ & $30.417 \pm 3.461^{\mathrm{c}, \mathrm{e}}$
\end{tabular}

Abbreviations: EPA, eicosapentaenoic acid; IL, interleukin; TNF, tumor necrosis factor.

${ }^{a} \mathrm{p}<0.05$.

${ }^{b} p<0.01$

${ }^{c} \mathrm{p}<0.001$ compared with the control group.

${ }^{d} p<0.05$

${ }^{e} \mathrm{p}<0.01$

${ }^{f} \mathrm{p}<0.001$ compared with the $25-\mu M$ Wogonin group.

${ }^{g} \mathrm{p}<0.05$.

$\& \& p<0.01$.

$\& \& \& p<0.001$ compared with the $100-\mu M$ Wogonin group.

This study has analyzed the molecular mechanism of $S$. baicalensis Georgi in gliomas by means of network pharmacology. The results revealed 85 targets of Wogonin acting with glioma. The results of the GO annotation and KEGG pathway analysis showed that these target genes were involved in the binding of phosphatidylinositol phosphokinase (to activate chemokines) and L-amino acid translocation (to activate amino acid translocation and thus activate and reverse the transport. The regulatory pathway involves the PI3K-Akt signaling pathway, the Rap1 signaling pathway, and the IL-17 signaling pathway. These results suggest that Wogonin could affect gliomas through the above regulatory pathways. Further research found that important target genes are concentrated in PI3K-Akt and IL-17 signaling pathways. We selected apoptotic factors such as caspase-3, Bad, and Bcl-2 and inflammatory factors such as IL- $1 \beta$, TNF- $\alpha$, and IL- 6 for further experimental verification.

Glioma is a common and destructive brain tumor that usually occurs in the glial cells of the brain or spine. Immunological studies have established the importance of the tumor microenvironment as a driver of tumor development. Inflammatory mediators such as IL$1 \beta$ released by monocytes regulate the transcription network needed for malignant cell growth (Tong et al., 2019). The uncontrolled expression of IL-6 in the central nervous system is usually closely related to the onset of neurodegenerative diseases and gliomas (Spooren et al., 2011). TNF- $\alpha$ is an initiator of inflammatory responses and has multipotent pro-inflammatory and neurotoxic effects (Tyrinova et al., 2018). The results of this study showed that $25,100,200 \mu \mathrm{M}$ of Wogonin inhibited the levels of these inflammatory factors in U251 glioma cells, and the IL-1 and IL-6 levels decreased in a dose-dependent manner with the increase in Wogonin concentration.

Wogonin, a monomer component extracted from $S$. baicalensis Georgi, is a flavonoid with a relative molecular weight of 284.26 and a molecular formula of $\mathrm{C}_{16} \mathrm{H}_{12} \mathrm{O}_{5}$. In vivo and in vitro analysis found that Wogonin can reduce the growth and metastasis of leukemia, liver cancer, colon cancer, breast cancer, and other malignant tumors (Huynh et al., 2017). Wogonin also strongly hinders the proliferation of U251 and U87 cells in glioma and induces their apoptosis (Tsai et al.,
TABLE 2 | The relative expression of caspase-3/cleaved-caspase-3, Bad, and $\mathrm{BCl}-2$ proteins.

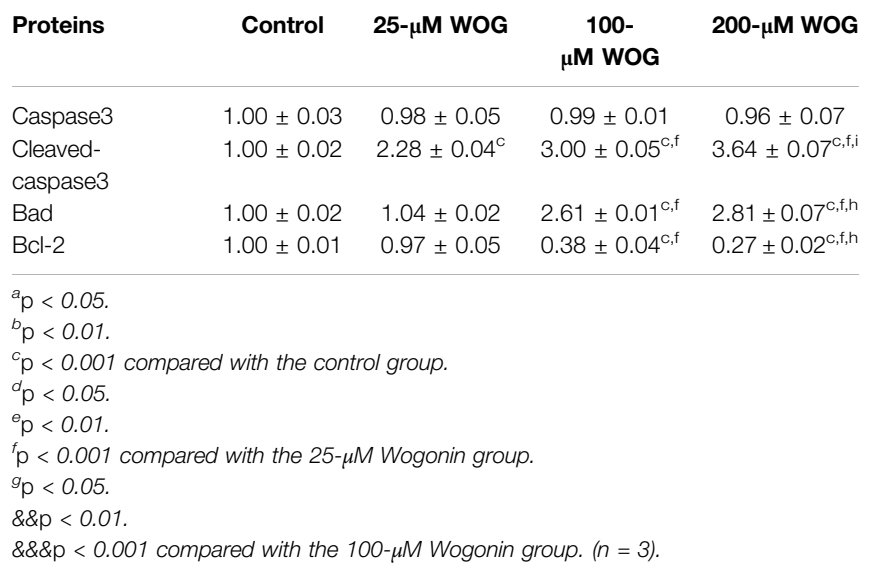

2012). (Wang et al., 2013) extracted Wogonin from $S$. baicalensis and found that Wogonin can inhibit the proliferation of glioma cells and induce G0/G1 stagnation in a dose-dependent manner. The expression of G1-phase cyclin D1 and cyclin-dependent kinases 2 and 4 was significantly decreased, and the cyclin-inhibiting protein P27 was overexpressed. The mechanism of promoting glioma differentiation might be the inhibition of glycogen synthase kinase-3/-light-chain protein pathway (Zhao et al., 2018). suggested that Wogonin could induce the phosphorylation and acetylation of $\mathrm{p} 53$, inhibit mouse double minute 2 homolog expression, and enhance p53 stability, thus suggesting Wogonin's wide range of antitumor effects. Another study on the effects of Wogonin on the proliferation and invasion of U87 glioma cells and its related mechanisms showed that Wogonin has the ability to inhibit U87 cell proliferation and invasion, decrease ezrin protein Bcl-2 and phosphorylated ezrin protein levels, and significantly increase Bax protein expression and the apoptosis index. The authors speculated that Wogonin might inhibit the antiapoptotic factor and promote the apoptosis-inducing factor, thereby inducing glioma cell apoptosis (Zeng and Liu, 2015). Bcl-2 is a classical anti-apoptotic protein in highly homologous proteins and plays an important regulatory role in the process of cell apoptosis (Wang et al., 2018). The downstream factor Bad is a target proapoptotic gene that regulates cell apoptosis and survival. Caspase-3 is the central link in the signal transduction process of apoptosis, and the detection of activated caspase- 3 can reflect the process of apoptosis (Shen et al., 2016). In this study, we detected the apoptotic factor levels using Western blotting, and the results showed that cleaved caspase-3 expression was significantly increased and was dosedependent on Wogonin. The expression of $\mathrm{Bad}$ and $\mathrm{Bcl}-2$ with $25 \mu \mathrm{M}$ of Wogonin has remained unchanged; however, when the Wogonin dose was $100 \mu \mathrm{M}$, the expression of Bad and Bcl-2 changed significantly (Bad was significantly upregulated, while Bcl-2 was significantly decreased) and was dosedependent on Wogonin. 
In conclusion, we speculate that Wogonin might promote glioma cell apoptosis by upregulating Bad gene expression and cleaved caspase-3 gene activation and by downregulating Bcl-2 expression. Wogonin also inhibits the progression of gliomas by downregulating the secretion of the inflammatory cytokines TNF- $\alpha$, IL- 6 , and IL- $1 \beta$. To provide a theoretical basis for clinical treatment, further in-depth experimental studies are needed to explore the specific molecular mechanisms of Wogonin's action on gliomas.

\section{DATA AVAILABILITY STATEMENT}

The original contributions presented in the study are included in the article/Supplementary Material, further inquiries can be directed to the corresponding author.

\section{REFERENCES}

Aquino, D., Gioppo, A., Finocchiaro, G., Maria Grazia, B., and Valeria, C. (2017). MRI in glioma immunotherapy: evidence, pitfalls, and perspectives[J]. J. Immunol. Res. 2017, 5813951. doi:10.1155/2017/5813951

Fan, X., Chai, L., Zhang, H., Wang, Y., Zhang, B., and Gao, X. (2015). Borneol depresses P-glycoprotein function by a NF- $\mathrm{KB}$ signaling mediated mechanism in a blood brain barrier in Vitro model. Ijms 16 (11), 27576-27588. doi:10.3390/ijms161126051

Galldiks, N., Kocher, M., and Langen, K.-J. (2017). Pseudoprogression after glioma therapy: an update. Expert Rev. Neurotherapeutics 17 (11), 1109-1115. doi:10. 1080/14737175.2017.1375405

Hervey-Jumper, S. L., and Berger, M. S. (2019). Insular glioma surgery: an evolution of thought and practice. J. Neurosurg. 130 (1), 9-16. doi:10.3171/2018.10.jns181519

Huynh, D. L., Sharma, N., Kumar Singh, A., Singh Sodhi, S., Zhang, J.-J., Mongre, R. K., et al. (2017). Anti-tumor activity of wogonin, an extract from Scutellaria baicalensis, through regulating different signaling pathways. Chin. J. Nat. Medicines 15 (1), 15-40. doi:10.1016/s1875-5364(17)30005-5

Jain, B., Raj, U., and Varadwaj, P. K. (2018). Drug target interplay: a network-based analysis of human diseases and the drug targets. Ctmc 18 (13), 1053-1061. doi:10.2174/1568026618666180719160922

Kyo, R., Nakahata, N., Sakakibara, I., Kubo, M., and Ohizumi, Y. (1998). Natural products: baicalin and baicalein, constituents of an important medicinal plant, inhibit intracellular $\mathrm{Ca} 2+$ elevation by reducing phospholipase $\mathrm{C}$ activity in $\mathrm{C} 6$ rat glioma cells. J. Pharm. Pharmacol. 50 (10), 1179-1182. doi:10.1111/j.20427158.1998.tb03331.x

Lee, S. W., Song, G. S., Kwon, C. H., and Kim, Y. K. (2005). Beneficial effect of flavonoid baicalein in cisplatin-induced cell death of human glioma cells[J]. Neurosci. Lett. 382 (1-2), 71-75. doi:10.1016/j.neulet.2005.03.005

Li, X., Xu, Y., Cui, H., Huang, T., Wang, D., Lian, B., et al. (2017). Prediction of synergistic anti-cancer drug combinations based on drug target network and drug induced gene expression profiles. Artif. Intelligence Med. 83, 35-43. doi:10. 1016/j.artmed.2017.05.008

Poornima, P., Kumar, J. D., Zhao, Q., Blunder, M., and Efferth, T. (2016). Network pharmacology of cancer: from understanding of complex interactomes to the design of multi-target specific therapeutics from nature. Pharmacol. Res. 111 (111), 290-302. doi:10.1016/j.phrs.2016.06.018

Scheck, A. C., Perry, K., Hank, N. C., and Clark, W. D. (2006). Anticancer activity of extracts derived from the mature roots of Scutellaria baicalensis on human malignant brain tumor cells. BMC Complement. Altern. Med. 6, 27. doi:10.1186/ 1472-6882-6-27

Shen, X., Burguillos, M. A., Osman, A. M., Frijhoff, J., Carrillo-Jiménez, A., Kanatani, S., et al. (2016). Glioma-induced inhibition of caspase-3 in microglia promotes a tumorsupportive phenotype. Nat. Immunol. 17 (11), 1282-1290. doi:10.1038/ni.3545

Sidders, B., Karlsson, A., Kitching, L., Torella, R., Karila, P., and Phelan, A. (2018). Network-based drug discovery: coupling network pharmacology with phenotypic screening for neuronal excitability. J. Mol. Biol. 430 (18 Pt A), 3005-3015. doi:10.1016/j.jmb.2018.07.016

\section{AUTHOR CONTRIBUTIONS}

$\mathrm{ZW}, \mathrm{XM}, \mathrm{CC}$, and JZ participated in the research design. ZW, LC, and ZS carried out the experiment. ZL, YZ, WJ, YL, FS, and JZ performed the data analysis. ZW, LC, X M, and CC wrote the manuscript.

\section{FUNDING}

This work was supported by the Science and Technology Research in Henan Province (202102310463). This work was supported by key project at central government level: The ability establishment of sustainable use for valuable Chinese medicine resources (2060302).

Spooren, A., Mestdagh, P., Rondou, P., Kolmus, K., Haegeman, G., and Gerlo, S. (2011). IL-1 $\beta$ potently stabilizes IL-6 mRNA in human astrocytes. Biochem. Pharmacol. 81 (8), 1004-1015. doi:10.1016/j.bcp.2011.01.019

Tong, L., Xie, C., Wei, Y., et al. (2019). Antitumor effects of berberine on Gliomas via inactivation of caspase-1-Mediated IL-1 $\beta$ and IL-18 release [J]. Front. Oncol. 9, 364. doi:10.3389/fonc.2019.00364

Tsai, C.-F., Yeh, W.-L., Huang, S. M., Tan, T.-W., and Lu, D.-Y. (2012). Wogonin induces reactive oxygen species production and cell apoptosis in human glioma cancer cells. Ijms 13 (8), 9877-9892. doi:10.3390/ijms13089877

Tu, Y. (2011). The discovery of artemisinin (qinghaosu) and gifts from Chinese medicine. Nat. Med. 17 (10), 1217-1220. doi:10.1038/nm.2471

Tyrinova, T., Leplina, O., Mishinov, S., Tikhonova, M., Kalinovskiy, A., Chernov, S., et al. (2018). Defective dendritic cell cytotoxic activity of high-grade glioma patients' results from the low expression of membrane TNFa and can Be CorrectedIn vitroby treatment with recombinant IL-2 or exogenic double-stranded DNA. J. Interferon Cytokine Res. 38 (7), 298-310. doi:10.1089/jir.2017.0084

Valentovic, M. A. (2018). Evaluation of resveratrol in cancer patients and experimental models. Adv. Cancer Res. 137, 171-188. doi:10.1016/bs.acr.2017.11.006

Wang, N., Zhang, Q., Luo, L., Ning, B., and Fang, Y. (2018). $\beta$-asarone inhibited cell growth and promoted autophagy via P53/Bcl-2/Bclin-1 and P53/AMPK/ mTOR pathways in Human Glioma U251 cells. J. Cel Physiol 233 (3), 2434-2443. doi:10.1002/jcp.26118

Wang, Y., Zhang, Y., Qian, C., Cai, M., Li, Y., Li, Z., et al. (2013). GSK3 $\beta / \beta$-catenin signaling is correlated with the differentiation of glioma cells induced by wogonin. Toxicol. Lett. 222 (2), 212-223. doi:10.1016/j.toxlet.2013.07.013

Wesseling, P., and Capper, D. (2018). WHO 2016 Classification of gliomas. Neuropathol. Appl. Neurobiol. 44 (2), 139-150. doi:10.1111/nan.12432

Zeng, Z., and Liu, H. (2015). [Inhibitory effect of wogonin on the proliferation and invasion of glioblastoma U87 cells and related mechanism]. Xi Bao Yu Fen Zi Mian Yi Xue Za Zhi 31 (3), 302-306. PMID:25744831

Zhang, Z., Lv, J., Lei, X., Li, S., Zhang, Y., Meng, L., et al. (2014). Baicalein reduces the invasion of glioma cells via reducing the activity of p38 signaling pathway[J]. PLoS One 9 (2), e90318. doi:10.1371/journal.pone.0090318

Zhao, Y., Zhang, L., Wu, Y., Dai, Q., Zhou, Y., Li, Z., et al. (2018). Selective antitumor activity of wogonin targeting the Warburg effect through stablizing p53. Pharmacol. Res. 135, 49-59. doi:10.1016/j.phrs.2018.07.011

Conflict of Interest: The authors declare that the research was conducted in the absence of any commercial or financial relationships that could be construed as a potential conflict of interest.

Copyright (c) 2021 Wang, Cheng, Shang, Li, Zhao, Jin, Li, Su, Mao, Chen and Zhang. This is an open-access article distributed under the terms of the Creative Commons Attribution License (CC BY). The use, distribution or reproduction in other forums is permitted, provided the original author(s) and the copyright owner(s) are credited and that the original publication in this journal is cited, in accordance with accepted academic practice. No use, distribution or reproduction is permitted which does not comply with these terms. 